METZLER LEXIKON

CHRISTLICHER DENKER 


\title{
METZLER LEXIKON CHRISTLICHER DENKER
}

\author{
700 Autorinnen und Autoren \\ von den Anfängen des Christentums \\ bis zur Gegenwart
}

herausgegeben von

Markus Vinzent

unter Mitarbeit von

Ulrich Volp und Ulrike Lange

Verlag J.B. Metzler

Stuttgart · Weimar 


\title{
Inhalt
}

\author{
Vorwort V \\ Artikel A-Z I \\ Verzeichnis der Mitarbeiterinnen und Mitarbeiter 762 \\ Abkürzungsverzeichnis 770 \\ Register 776 \\ Artikelverzeichnis 8I5
}

Die Deutsche Bibliothek - CIP-Einheitsaufnahme

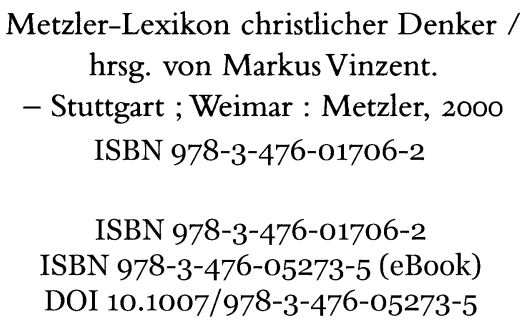

Dieses Werk einschließlich aller seiner Teile ist urheberrechtlich geschützt. Jede Verwertung außerhalb der engen Grenzen des Urheberrechtsgesetzes ist ohne Zustimmung des Verlages unzulässig und strafbar. Das gilt insbesondere für Vervielfältigungen, Übersetzungen, Mikroverfilmungen und die Einspeicherung und Verarbeitung in elektronischen Systemen.

(C) 2000 Springer-Verlag GmbH Deutschland

Ursprünglich erschienen bei J.B. Metzlersche Verlagsbuchhandlung

und Carl Ernst Poeschel Verlag GmbH in Stuttgart 2000

www.metzlerverlag.de

info@metzlerverlag.de 


\section{Vorwort}

Am Anfang des Metzler Lexikon christlicher Denker ein Wort des Dankes: den Wissenschaftskolleginnen und -kollegen; denn es gibt das Kollegium. Nur etwa ein Prozent der vom Herausgeber angeschriebenen, mit anderen Verpflichtungen wie Vorlesungen, Forschungsarbeiten, vielen anderen Lexikonartikeln, Verwaltung u.a. aus- und überlasteten Wissenschaftlerinnen und Wissenschaftlern hat eine Mitarbeit an diesem Werk - und dann fast immer mit bestens zu verstehenden Gründen abgelehnt. Eine große Zahl hat nicht nur pflichtgemäß den (oder die) angebotenen Artikel übernommen, sondern sich als aktive Kritiker am Umfang des vom Herausgeber avisierten Personenkreises (sowohl der Lemmata wie deren Autorinnen und Autoren) beteiligt. Am Ende hätte ein mehrbändiges Werk erscheinen können, wäre nicht der eingeschränkte Umfang notwendig für einen vertretbaren Preis. Ein Dank sei auch den Autorinnen und Autoren gesagt, die einige der geeigneten im Metzler Lexikon antiker Autoren erschienenen Artikel zurVerfügung gestellt oder diese für den Neuabdruck hier z. T. überarbeitet haben. Auch dem Verlag und seinem unermüdlichen Lektor Oliver Schütze sei gedankt; kritisch mit Sachverstand begleitend und aufmunternd hat er das Projekt von Anfang an auch inhaltlich gefördert. Die Idee und der Anstoß zu diesem Lexikon stammen von meinem Freund und Historikerkollegen, Herrn Hochschuldozenten PD Dr. Stefan Rebenich (Mannheim), dem ich hierfür herzlich danke. Die praktische Logistik für das Vorhaben gewann erheblich, nachdem an der Universität Birmingham die wissenschaftlichen Assistenten, Herr Ulrich Volp und Frau Ulrike Lange, in das Projekt einstiegen. Ihrer Akribie und Mühe bei diversen Recherchen und dem Bewältigen des nicht abreißenden Stroms an Korrespondenzen ist es zu verdanken, daß das Werk zum vorgesehenen Zeitpunkt abgeschlossen werden konnte. Schließlich hat Herr Ulrich Volp eigenverantwortlich den Index und das Mitarbeiterverzeichnis erstellt und Übersetzungen von englischsprachigen Artikeln angefertigt. Mit ihm konnte ich auch Fragen diskutieren, wie die einzelnen Lemmata alphabetisch zu ordnen seien (wir verständigten uns auf einen Kompromiß: bis zur Reformationszeit werden die Lemmata - soweit sinnvoll - nach den Vornamen angeführt). Ganz am Ende ist mir auch meine Frau, Dr. Jutta Vinzent, wiederholt zur Hand gegangen, nachdem sie die gesamte Zeit über die Herausgabe begleitend unterstützt hatte.

Die Verantwortung für die Schwächen dieses Lexikons liegen alleine bei mir. In einer Zeit der Interaktivität freue ich mich, möglichst viele Anregungen und Kritiken zu erhalten (markusvinzent@hotmail.com), die einer eventuellen, erweiterten zweiten Auflage zugute kommen können.

Sind Denken und christlicher Glaube vereinbar? Sowohl Philosophen als auch Theologen und "Gläubige" derVergangenheit und Gegenwart haben eine Kompatibilität beider unterschiedlichen Größen wieder und wieder bestritten, oft mit guten Gründen, wie es scheint. Diese basieren auf dem Widerspruch (oder zumindest auf einer natürlichen Konkurrenz) von Wissen und Glauben, von Vernunft und Vertrauen. Von nichtchristlicher Seite aus wird kritisch auf die Verquickung von 
Glauben und Vertrauen mit Institutionen wie Kirchen, Hierarchien, Dogmen und Gemeinden hingewiesen. Manche Christen lehnen einen inneren Bezug von Vernunft und Glauben ab, weil sie in der grundsätzlich freien Vorstellung und im kritischen Selbstbewußtsein des Menschen den Ausdruck eines ungebändigten Individualismus und eine anthropologische Zentrierung und Hybris erkennen, die dem auf Gemeinschaft angelegten Christentum und dem in ihm vertretenen transzendenten Gottesbegriff widerspreche, der alles im Sündenfall gebrochene Menschliche relativiere.

Auch wenn man das Verhältnis von Denken und christlichem Glauben historisch betrachtet, wird man zunächst eher auf deren Unvereinbarkeit stoßen. Faktum ist, daß die geistige Bewußtwerdung des Menschen und die Entwicklung einer ausgesprochenen Philosophie Jahrhunderte vor dem Auftreten Jesu von Nazareth und der Entstehung des Christentums einsetzte und - entgegen späteren jüdischen und christlichen Geschichtsklitterungen - von jüdisch-christlichen Traditionen lange Zeit unabhängig war. Gewiß verweist die Geschichte in Kleinasien und Europa, später auch in weiten Teilen der übrigen Welt darauf, daß die historische Entwicklung des menschlichen Geistes nicht zu verstehen ist, wenn der Einfluß christlicher Denkkategorien (nicht anders als hellenistischer, jüdischer, islamischer und solcher anderer alter und neuer Religionen und Kulturen) ausgeblendet wird.

Gleichwohl will das vorliegende Metzler Lexikon christlicher Denker nicht aus phänomenologisch-faktischen Gründen die verästelten Auswirkungen des Christentums auf die Entwicklung der Geistesgeschichte dokumentieren oder auch umgekehrt die spannungsgeladene und unbeendete Odyssee zwischen Philosophie und Theologie nachzeichnen, so aufschlußreich beide Unternehmen auch wären. Es konzentriert sich vielmehr darauf, die Herausforderungen zu verdeutlichen, die christliche Kategorien an überkommenes Denken stellen, und umgekehrt die Anfragen aufzuzeigen, die der ewig kritische menschliche Geist an das Christentum heranträgt. Um bei letzterem zu beginnen: Es bedurfte nicht erst der Aufklärung, daß sich das Christentum als ethische Philosophie begriff. Gerade in den ersten Jahrhunderten - die darum in diesem Lexikon etwas ausführlicher berücksichtigt sind - meinen Gelehrte, die sich dem Christentum anschließen, sie hätten die "wahre Philosophie" umarmt. Doch ist diese Liebe zur Weisheit nicht mehr dieselbe wie die der vorchristlichen Zeit. Der menschliche Geist steht - selbst als göttlicher Reflex - nicht mehr einsam dirigierend auf dem Podest. Ihm zur Seite getreten ist der Körper mit all seinen sozialen Verflechtungen. Bald kommt mit Augustinus von Hippo der Wille des Menschen hinzu, der sowohl der menschlichen Vernunft wie auch der leiblichen Dominanz den Rang abläuft. Doch gerade der Wille setzt sich in eine eigentümliche Spannung zwischen Selbstverliebtheit und Gottesdistanz auf der einen Seite und einem auf das menschliche und göttliche Du bezogenen Eros auf der anderen. Der göttliche Geist wiederum residiert ebenfalls nicht mehr alleine. Er wird unlösbar gepaart mit dem Menschen, in kreatürlicher Verbindung in Christus, in göttlicher Einheit in der Trinität. Das Paradox der Verbindung von Gott und Mensch wird im Christentum statuiert und erklärt, aber nicht erläutert.

Woher kommt der Anspruch im Christentum, daß die religiöse Praxis einer geistigen Herausforderung bedarf? Es scheint mir das Resultat aus mehrerlei Voraus- 
setzungen. Von philosophischer Seite war die Grundlage für eine philosophische Religion gelegt durch das religiös fundierte Denken in der antiken, speziell kaiserzeitlichen pythagoreisch-platonischen, aristotelischen wie stoischen Philosophie. Die Modephilosophie der Kaiserzeit schloß den Gottes- und Wahrheitsbegriff ebenso ein wie eine den Menschen und seine Welt umfassende Ethik. Noch bevor das Christentum existierte, hatte die griechisch-römische Denkkultur tief die jüdische Glaubens- und Vorstellungswelt durchdrungen, so daß heute eine Scheidung von jüdisch-christlichem Vertrauen (hebr. emuna) und griechischem Glauben (gr. pistis) von vornherein zum Scheitern verurteilt ist. Umgekehrt war der Raum, in dem das Christentum groß wurde, kein glaubensfreier und selten ein atheistischer Denkhorizont, er war aber auch kein unkritisch frommer, unreflektiert fundamentalistischer Boden. Das Christentum hat sich, ja konnte sich expansiv nur dadurch entwickeln, indem es diese vorgefundene innere Verbindung von Philosophie und Religion annahm und innovativ fortschrieb. $\mathrm{Zu}$ nutzen kam ihm, daß die zeitgenössische Philosophie aufgrund der Rückwirkung jüdischer Einflüsse eine deutlich monotheistische Ausprägung entwickelt hatte, der jüdische Monotheismus aber seinerseits aufgrund aufgenommener philosophischer Spekulationen längst nicht monistisch, sondern durchaus polytheistisch gefärbt war. Das im frühen Christentum dominierende griechischsprachige "volksphilosophisch"ethisch orientierte hellenistische Rabbinertum wiederum übte früh auf die schriftlich gefaßte Interpretation der Person Jesu maßgeblichen Einfluß aus. Von der ersten Generation ab war das Christentum innerhalb eines viele Kulturen, Sprachen, Völker, Länder und Kontinente umfassenden Roms auf die (volks-)philosophische Fährte gesetzt.

Heute, im beginnenden $2 \mathrm{I}$. Jahrhundert, das den weiteren Aufstieg der toleranten Multikulturalität erleben mag, wird ein kreatives Zusammenleben der verschiedenen Denk-, Lebens- und Glaubensweisen nur möglich sein, wenn ein historischsensibles Gespür für die in Vergangenheit und Gegenwart bedeutsamen geistigen Entwürfe wächst. Aus diesem heraus ist die Zukunft zu entwickeln, die sich gerade erst andeutet. Auch wenn bereits zu Anfang des 20. Jahrhunderts die innerchristlich ökumenische Frage wach geworden war und nach der Tragödie eines Zweiten Weltkrieges das Zusammenleben der Religionen und Kulturen mit ihren verschiedenen Ethikvorstellungen offener thematisiert wurde, blieb bei der Betrachtung von geistigen Entwürfen der Vergangenheit bislang weitgehend außer Acht, welche sozioanthropologische und religionshistorische Herausforderung ein nichtreligiöses und individuoreligiöses Umfeld darstellt. Eine nüchterne, verstehenwollende, mehr als duldend-tolerante, ja empathische und kreative Bemühung um den Nichtglauben des Anderen bzw. dessen Eigenreligion gilt es erst zu entwickeln. Notwendig wird für eine global vernetzte Menschheit, die immer stärker in der Spannung zwischen Religionen und Nichtreligion, zwischen ethischen Traditionen und individuellen Moralvorstellungen lebt, ein erfindungsreiches Wissensmanagement und eine Erfahrenspädagogik von Religions- und Ethikkenntnissen, die nicht nur von und für Insider gemacht und gedacht sind, sondern aus denen Referenzpunkte entwickelt werden, an denen gerade einander sich Fremde gemeinsam orientieren können. Während im deutschsprachigen Bereich christliche Theologie 
und Religionsunterricht noch konfessionell in Konkurrenz zu Ethik und Lebenskunde stehen und aus Angst, auf dem freien Markt unterzugehen, sich im historisch angestammten und verfassungsmäßig abgesicherten konfessionellen christlichen Korsett einzwängend absichern, boomt die Nachfrage nach wissenschaftlich verläßlicher interreligiöser Forschung und aufgeschlossen kritischem Umgang mit Religion, Religionen und Ethik. Immer öfter sucht die Gegenwart Antworten auf die Frage, wie sich jenseits von Religion und Glaube, ohne Gottesdienst und Verehrung (auch Selbstverehrung) sinnvoll leben läßt.

Längst sind außerhalb des einst kolonialmächtig-traditionellen Europa Neuansätze für die Untersuchung auch des Christentums entstanden, das dessen Anspruch auf Wahrheit ernst nehmen kann, ohne sich diesen zueigen zu machen. Die Erforschung christlicher Traditionen durch Nichttheologen, Nichtchristen, Nichtglaubende oder Atheisten ist - entgegen einer bis heute in Insiderkreisen vertretenen Auffassung - möglich und erbringt wichtige Einsichten für den multikulturellen Umgang. Durch die Ent-Europäisierung und notwendige Globalisierung der Bildung ist die Zeit eigentlich abgelaufen, in der staatliche oder religiöse Institutionen den Zugang zu Religionswissen kanalisieren und kontrollieren, zumal das Bedürfnis nach diesem Wissen innerhalb und außerhalb von Konfessionen und Religionen weiterhin wächst.

Eine Antwort auf das interessierte Fragen nach den vom Christentum angeregten geistigen und oft ethisch-praktischen Entwürfen wird mit dem vorliegenden Metzler Lexikon christlicher Denker (und Denkerinnen) gegeben. Die Autorinnen und Autoren kommen aus den verschiedensten auch nichtdeutschsprachigen Ländern, Traditionen, Konfessionen und weltanschaulichen Verortungen. Es wurde bei der Auswahl weder der Autorinnen und Autoren noch der $\mathrm{zu}$ behandelnden Persönlichkeiten auf einen konfessionellen Proporz geachtet. Vertraut mit katholischen und vor allem protestantischen Traditionen, vor allem der älteren Geschichte des Christentums, basiert der Denkansatz des Herausgebers auf einer Form der game theory (Spieltheorie), die Denken und Glauben, Philosophien, Konfessionen und Religionen (darunter auch das Christentum) als grundlegende - und darum immer tiefer $\mathrm{zu}$ verstehende - Regeln menschlichen Denkens und (Zusammen-)lebens begreift. Wie bei einem Spiel aber - sei es das einfache Würfeln oder etwa die hochkomplexe Börse - bilden Anweisungen lediglich die notwendigen Bedingungen, um den eigentlichen Inhalt, nämlich das existentielle Spiel, zu ermöglichen. Spieltheoretischer Untersuchungsgegenstand ist immer das noch Unbestimmte und jeweils Kreative, das diese Welt und in ihr Leben wie Tod bieten. Von Natur aus lassen sich Kreativität und innovative Ergebnisse nur in ihren Voraussetzungen und in den durch sie eröffneten Möglichkeiten beschreiben. Als Ergebnisse bleiben sie vorläufig. Gleich welcher der Denkansätze im Christentum, der Philosophie oder einer anderen Religion vor Augen treten, gemessen an der größeren Fülle der zu denkenden Möglichkeiten, ist jeder begrenzt. Auch der tolerante Relativismus greift zu kurz. Er übersieht die mögliche Interaktion der verschiedenen Konfessionen, Religionen, Weltanschauungen, Philosophien und ihrer Vertreter. Das Kreative nicht als mythologisch unbestimmbare Größe gedacht, sondern als letztherausfor- 
dernde Skepsis gegenüber allem Erreichten - umfaßt Denken und Glauben und Nichtglauben, Philosophie und Theologie und Atheologie. Als christlicher Denker oder Theologe gilt nicht schon diejenige Person, die das Wort "Gott" häufig in den Mund nimmt oder zwischen Zeilen bannt, indem sie voraussetzt, was jedes Denken als selbstkritischer Reflex immer erst in Frage stellt. Vielmehr sind diejenigen Entwürfe von Interesse, die die eigene Perspektive in unerforschte Gebiete hinein verlängern oder sie radikal in Frage stellen. Wenn Denken und Christentum in diesem Lexikon zusammengebracht und bedacht werden, weil Denken mehr als Theorie und Christentum mehr als Frömmigkeit und Gottesglauben sein kann (geschweige denn die später in ihm entwickelte Lehre von Inkarnation und Trinität), so entspricht dieser Weite, daß hier Personen vor Augen treten, die in der Geschichte das Christentum in seinen Traditionen erhellt, öfter aber solche, die ihm ihr eigenes unverwechselbares Gepräge gegeben haben. Dabei war für die Auswahl unerheblich, ob die Entwürfe zeitgebunden oder dauerhaft waren. Aus der natürlichen Spannung zwischen Spontaneität und sich institutionalisierendem Regelwerk ergibt sich, daß die Zahl der die Grenzen von staatlichen Gebilden, Kirchen und religiösen Gemeinschaften überschreitenden Denkerinnen und Denkern entsprechend groß ist und bisweilen Personen und ihr Lebenswerk vorgestellt werden, die in einem Theologenlexikon kaum Aufnahme gefunden hätten (z. B. Schopenhauer). Aus demselben Grund wird man Personen vermissen, die in jedem Kirchenlexikon zu finden sind. Es erklärt sich aus dem gewählten Ansatz auch die Aufnahme der vielen in den eigenen Ländern und Kirchen verfolgten und nicht selten zu Tode gekommenen "Häretiker" und "Häretikerinnen". Auch die Grenze der »Theologie» (eine geschichtlich gesehen relativ junge Kategorie: noch bis zum Ende der Spätantike verstanden sich christliche Denker als Philosophen) wurde häufig überschritten, um die Breite der Denk- und vor allem Praxisansätze aufzunehmen. Um spezifisch christliche innovative Entwürfe aufzuspüren, werden Bibelauslegung, historische Forschung, praktisch gelebte Ansätze und politisches Wirken vorgestellt. Gerade mit der Verbreiterung der "philosophia" in einen (allerdings oft schulmäßig tradierten) Fächerkanon hat das Christentum die theoretische Enge und Stringenz der antiken paganen Ethik und Philosophie gesprengt. Vergleicht man etwa das Metzler Philosophen Lexikon mit dem vorliegenden Werk, wird die größere Zahl kleinerer Artikel auffallen. Sie soll nicht den Kurzschluß nahelegen, daß es im Christentum keine Denkerinnen und Denker gegeben habe, denen wie in der Philosophie lange Ausführungen angemessen gewesen wären. Im Gegenteil, nimmt man zur Kenntnis, daß spätestens vom Ende der Spätantike bis zur Aufklärung und teilweise darüber hinaus die Geschichte der Philosophie weitgehend eine solche des christlichen Denkens ist, christliches Denken aber, wie viele der Einträge zeigen, von Anfang an inhärent und explizit meist auch politisch-praktisch orientiert, ja manchmal nur an der ethischen Praxis ablesbar ist, dann erklärt sich die größere Anzahl der Personen in diesem Lexikon. Auch wenn dennoch für die Zeit bis ins I9. Jahrhundert weithin die bedeutenden, auch im Metzler Philosophen Lexikon Verzeichneten, aufgenommen wurden, mußte für das 20. Jahrhundert allein aus Platzgründen auf viele Namen verzichtet werden (aufgenommen wurden fast ausschließlich bereits Verstorbene), die inhaltlich in das Konzept dieses Lexikons 
gehört hätten (z.B. K. Jaspers, M. Heidegger u.a.). Gegebenenfalls läßt sich bei einer erweiterten Zweitauflage gerade dieses Manko beheben.

Markus Vinzent

Birmingham, im August 2000 